\title{
ESSENTIALLY TRIANGULAR ALGEBRAS ${ }^{1}$
}

\author{
J. A. ERDOS AND A. HOPENWASSER
}

\begin{abstract}
If $\mathcal{N}$ is a nest, then the set of all bounded linear operators $T$ such that $T P-P T P$ is compact for all $P$ in $\mathcal{N}$ is the essentially triangular algebra associated with $\mathcal{N}$. Put another way, essentially triangular algebras are the inverse images under the Calkin map of nest-subalgebras of the Calkin algebra. J. Deddens has characterized nest algebras in terms of operators whose half-orbits under similarity by a positive invertible operator are bounded in norm. This paper, by substituting essential norms for norms, provides a related characterization of essentially triangular algebras.
\end{abstract}

There are three different ways one could define "nest algebra": as the set of all operators which leave invariant a given chain of projections on Hilbert space; as the set of analytic operators with respect to some ultraweakly continuous oneparameter family of inner automorphisms of $B(\mathcal{H})$; and as the set of all operators which have a norm bounded half-orbit under the similarity transform induced by a given positive invertible operator. The first definition is the original one due to Ringrose [10]; the second is the Loebl-Muhly characterization of nest algebras [8]; the third is Deddens' characterization [4]. Deddens' original proof of his description of nest algebras is based on the Loebl-Muhly characterization, which in turn uses Arveson's theory of spectral subspaces. An elementary proof of the Deddens characterization may be found in [5]. In this note we extend those arguments to apply to essentially bounded half-orbits and to essentially triangular algebras (i.e., those operators which leave a chain of projections invariant modulo the compact operators).

Compact perturbations of nest algebras play a significant role in the theory of nest algebras. This is particularly evident in the work on similarities and multiplicity by Andersen [1, 2], Larson [7], and Davidson [3]. At least tacitly, compact perturbations lead to subalgebras of the Calkin algebra. If a nest of projections in the Calkin algebra is the image under the canonical quotient map of a nest in $B(\mathcal{H})$, then the associated nest algebra in the Calkin algebra is the image of an essentially triangular subalgebra of $B(\not H)$. Thus, the Deddens condition stated with the essential norm in place of the usual operator norm is related to nest-subalgebras of the Calkin algebra. Nest-subalgebras of other $C^{*}$-algebras have been studied recently by S. Power [9].

Fix notation as follows: $\not{H}$ will denote a separable Hilbert space; $B(\not H)$, the set of bounded linear operators on $H ; K$, the set of compact operators, and $Q=$ $Q(\mathcal{H})=B(\mathscr{H}) / \mathcal{K}$, the Calkin algebra. If $\mathcal{N}$ is a nest of projections in $B(\mathcal{H})$, then, as usual, $\operatorname{Alg} \mathcal{N}=\{T \in B(\mathcal{H}): T P=P T P$ for all $P \in \mathcal{N}\}$. Let $\operatorname{ET}(\mathcal{N})=\{T \in$

Received by the editors February 11, 1985.

1980 Mathematics Subject Classification. Primary 47D25.

${ }^{1}$ This research was supported by grants from the SERC (UK) and the NSF (USA). 
$B(\mathcal{H}): T P-P T P \in \mathcal{K}$ for all $P \in \mathcal{N}\} . \operatorname{ET}(\mathcal{N})$ is called the essentially triangular algebra associated with $\mathcal{N}$.

Let $A$ be a positive invertible operator in $B(\mathscr{H})$ and let $E(\cdot)$ be the associated projection-valued spectral measure for $A$ (so $A=\int_{\mathbf{R}} \lambda d E(\lambda)$ ). Define the spectral nest of $A$ to be the family of all projections of either of the forms $E[0, t)$ or $E[0, t]$, for $t \geq 0$. A spectral nest is clearly closed in the strong operator topology and, in particular, complete as a sublattice of the full projection lattice in $B(\mathcal{H})$.

Let $\mathcal{N}$ be a nest and let $A$ be any positive invertible operator whose spectral nest is $\mathcal{N}$. (The existence of such operators is a routine matter.) Then Deddens' result states that $\operatorname{Alg} \mathcal{N}=\left\{T \in B(\mathcal{H})\right.$ : the sequence $\left\|A^{n} T A^{-n}\right\|, n=0,1,2, \ldots$, is bounded $\}$. We shall refer to $\left\{A^{n} T A^{-n}: n=0,1,2, \ldots\right\}$ as the half-orbit of $T$ under the action of $A$. Note that the norm-boundedness of the half-orbit of $T$ depends only on the choice of nest $\mathcal{N}$; it is independent of the choice of operator with spectral nest equal to $\mathcal{N}$. If we replace boundedness in norm by boundedness in essential norm, then the choice of the operator $A$ becomes significant.

The essential norm of an operator $T$ in $B(\mathcal{H})$ is given by $\|T\|_{e}=\inf \{\|T+K\|: K \in$ $K\}$. If $\pi: B(\mathcal{H}) \rightarrow \mathcal{Q}$ is the canonical quotient map of $B(\mathcal{H})$ onto the Calkin algebra, then $\|T\|_{e}=\|\pi(T)\|$. Fix a nest $\mathcal{N}$ and a positive invertible operator $A$ whose spectral nest is $\mathcal{N}$. Let $B(A)$ denote the set of all operators $T$ whose half-orbit is essentially bounded; i.e., $B(A)=\left\{T \in B(\not H)\right.$ : the sequence $\left\|A^{n} T A^{-n}\right\|_{e}, n=$ $0,1,2, \ldots$, is bounded $\}$. Our first task is to characterize $B(A)$.

For each $E>0$ in $\mathcal{N}$, define $\lambda_{E}=\|A E\|$; for each $E<I$ define $\mu_{E}=$ $\left\|A^{-1} E^{\perp}\right\|^{-1}$. Then $A E^{\perp} \geq \mu_{E} E^{\perp}$ and $\mu_{E}$ is the largest constant with this property. (One may think of $\mu_{E}$ as the "greatest lower bound" for $A$ on $E^{\perp}$.) Because $\mathcal{N}$ is the spectral nest for $A, \lambda_{E} \leq \mu_{E}$ for all $E \neq 0, I$. If $\lambda_{E}<\mu_{E}$, we say that $A$ has a jump at $E$. Since $\left\|A^{-1}\right\|^{-1}=\mu_{0} \leq \lambda_{E} \leq \mu_{E} \leq \lambda_{I}=\|A\|$, there are at most countably many jumps, and at most finitely many with $\mu_{E}-\lambda_{E}$ greater than some specified value. A fairly routine argument yields the fact that, given $\varepsilon>0$, there is a finite subnest $0=E_{0}<E_{1}<\cdots<E_{k}=I$ of $\mathcal{N}$ with the following two properties:

(i) if $A$ has a jump at $E$ with $\mu_{E}-\lambda_{E} \geq \varepsilon$, then $E=E_{i}$ for some $i=1,2, \ldots, k-1$,

(ii) $\lambda_{E_{i+1}}-\mu_{E_{i}}<\varepsilon$ for all $i=0,1, \ldots, k-1$.

One other remark is in order. Generally speaking, when $E<F$ we usually have $\lambda_{E}<\mu_{F}$. In one case an exception is possible: if $E$ has no immediate predecessor, $F$ has no immediate successor, and $E$ is the immediate predecessor of $F$, then we may possibly have $\|A E\|=\left\|A^{-1} F^{\perp}\right\|^{-1}$; i.e., $\lambda_{E}=\mu_{F}$. This common value is then an eigenvalue for $A$; the corresponding eigenspace is the range of $F-E$.

Let $J=\left\{(E, F) \mid E, F \in \mathcal{N}\right.$ and $\left.\lambda_{E}<\mu_{F}\right\}$. So, $J$ consists of all the pairs $(E, F)$ with $E<F, E \neq 0, F \neq I$ except the special pairs described above and also all the pairs $(E, E)$ for which $A$ has a jump at $E$. We are now in a position to characterize $B(A)$.

Proposition 1. Let $A$ be a positive invertible operator with spectral nest $\mathcal{N}$. Then $B(A)=\left\{T \in B(\mathcal{H}): F^{\perp} T E \in \mathcal{K}\right.$ for all $\left.(E, F) \in J\right\}$.

Proof. First suppose that $T \in B(A)$. Let $M$ be such that $\left\|A^{n} T A^{-n}\right\|_{e} \leq M$ for all $n=0,1,2, \ldots$ Suppose $(E, F) \in J$. Then $\|A E\|_{e} \leq\|A E\|=\lambda_{E}$ and 
$\left\|A^{-1} F^{\perp}\right\|_{e} \leq\left\|A^{-1} F^{\perp}\right\|=1 / \mu_{F}$. For each $n \geq 0$, we have

$$
\begin{aligned}
\left\|F^{\perp} T E\right\|_{e} & =\left\|F^{\perp} A^{-n} A^{n} T A^{-n} A^{n} E\right\|_{e} \\
& \leq\left\|A^{-n} F^{\perp}\right\|_{e}\left\|A^{n} T A^{-n}\right\|_{e}\left\|A^{n} E\right\|_{e} \leq\left(\lambda_{E} / \mu_{E}\right)^{n} M .
\end{aligned}
$$

Since $\lambda_{E} / \mu_{E}<1$, this implies that $\left\|F^{\perp} T E\right\|_{e}=0$, i.e., $F^{\perp} T E \in \mathcal{K}$.

Now assume that $F^{\perp} T E \in \mathcal{K}$ for all pairs $(E, F) \in J$. We shall prove that $T \in B(A)$ by showing that $\left\|A^{n} T A^{-n}\right\| \leq 7\|T\|_{e}$ for all $n \geq 0$. Fix $n$ once and for all. Choose $\varepsilon=\frac{1}{2} \mu_{0}\left(2^{1 / n}-1\right)$. The choice of $\varepsilon$ is dictated by the following property: If $\lambda$ and $\mu$ are any two numbers such that $\mu_{0} \leq \mu \leq \lambda \leq \mu+2 \varepsilon$, then $(\lambda / \mu)^{n}<2$. Now choose a finite subnest $0=E_{0}<E_{1}<\cdots<E_{k}=I$ of $\mathcal{N}$ so that $\lambda_{E_{i+1}}-\mu_{E_{i}}<\varepsilon$ for all $i=0,1, \ldots, k-1$ and so that whenever $A$ has a jump $E$ with $\mu_{E}-\lambda_{E} \geq \varepsilon$, then $E=E_{i}$ for some $i$. Let $\Delta_{i}=E_{i}-E_{i-1}, i=1,2, \ldots, k$.

Let $T_{0}=\sum_{i=1}^{k} \Delta_{i} T \Delta_{i}, T_{1}=\sum_{i=1}^{k-1} \Delta_{i+1} T \Delta_{i}$ and $R=T-T_{0}-T_{1}$. We shall show that $\left\|A^{n} T_{0} A^{-n}\right\|_{e} \leq 2\|T\|_{e},\left\|A^{n} T_{1} A^{-n}\right\|_{e} \leq 2\|T\|_{e}$ and $\left\|A^{n} R A^{-n}\right\|_{e} \leq$ $3\|T\|_{e}$. Since $A^{n} T A^{-n}=A^{n} R A^{-n}+A^{n} T_{0} A^{-n}+A^{n} T_{1} A^{-n}$, this will yield $\left\|A^{n} T A^{-n}\right\|_{e} \leq 7\|T\|_{e}$, as desired.

Since $A$ commutes with each $\Delta_{i}$, we have $\left\|A^{n} T_{0} A^{-n}\right\|_{e}=\max \left\|A^{n} \Delta_{i} T \Delta_{i} A^{-n}\right\|_{e}$ and $\left\|A^{n} T_{1} A^{-n}\right\|_{e}=\max \left\|A^{n} \Delta_{i+1} T \Delta_{i} A^{-n}\right\|_{e}$. For each $i=1,2, \ldots, k,\left\|A^{n} \Delta_{i}\right\|_{e} \leq$ $\left\|A^{n} \Delta_{i}\right\| \leq \lambda_{E_{i}}^{n}$ and $\left\|\Delta_{i} A^{-n}\right\|_{e} \leq\left\|\Delta_{i} A^{-n}\right\| \leq\left(1 / \mu_{E_{i-1}}\right)^{n}$. Further, by the choice of the partition, $\lambda_{E_{i}} \leq \mu_{E_{i-1}}+\varepsilon$, whence

$$
\left\|A^{n} \Delta_{i} T \Delta_{i} A^{-n}\right\|_{e} \leq\left(\lambda_{E_{i}} / \mu_{E_{i-1}}\right)^{n}\|T\|_{e} \leq 2\|T\|_{e} .
$$

From this, $\left\|A^{n} T_{0} A^{-n}\right\|_{e} \leq 2\|T\|_{e}$ is immediate. A slight variation will take care of $A^{n} T_{1} A^{-n}$. Let $H=\left\{i \mid \lambda_{E_{i}}=\mu_{E_{i}}\right\}$. If $i \notin H$, then $\left(E_{i}, E_{i}\right) \in J$ and $\Delta_{i+1} T \Delta_{i} \in \mathcal{K}$. If $i \in H$, then $\lambda_{E_{i}} \leq \mu_{E_{i-1}}+\varepsilon, \lambda_{E_{i}}=\mu_{E_{i}}$, and $\lambda_{E_{i+1}} \leq \mu_{E_{i}}+\varepsilon$, whence $\lambda_{E_{i+1}} \leq \mu_{E_{i-1}}+2 \varepsilon$. Therefore, for $i \in H$,

$$
\left\|A^{n} \Delta_{i+1} T \Delta_{i} A^{-n}\right\|_{e} \leq\left(\lambda_{E_{i+1}} / \mu_{E_{i-1}}\right)^{n}\|T\|_{e} \leq 2\|T\|_{e} .
$$

Since $\sum_{i \notin H} A^{n} \Delta_{i+1} T \Delta_{i} A^{-n} \in \mathcal{K}$, we obtain

$$
\begin{aligned}
\left\|A^{n} T_{1} A^{-n}\right\|_{e} & =\left\|\sum_{i \in H} A^{n} \Delta_{i+1} T \Delta_{i} A^{-n}\right\|_{e} \\
& =\max _{i \in H}\left\|A^{n} \Delta_{i+1} T \Delta_{i} A^{-n}\right\|_{e} \leq 2\|T\|_{e} .
\end{aligned}
$$

All that remains is to prove that $\left\|A^{n} R A^{-n}\right\|_{e} \leq 3\|T\|_{e}$. Let $N=\sum_{i<j} \Delta_{i} T \Delta_{j}$ and $K=\sum_{i \geq j+2} \Delta_{i} T \Delta_{j}$. Then $R=N+K, N \in \operatorname{Alg} \mathcal{N}$ and $K \in \mathcal{K}$. The inequality $\left\|A^{n} N A^{-n}\right\| \leq\|N\|$ for $N \in \operatorname{Alg} \mathcal{N}$ is proven in [5]. The exact same proof remains valid for essential norms, so we have $\left\|A^{n} N A^{-n}\right\|_{e} \leq\|N\|_{e}$. Since $K$ is compact, we then obtain the string of inequalities

$$
\begin{aligned}
\left\|A^{n} R A^{-n}\right\|_{e} & =\left\|A^{n} N A^{-n}\right\|_{e} \leq\|N\|_{e} \\
& =\|R\|_{e} \leq\|T\|_{e}+\left\|T_{0}\right\|_{e}+\left\|T_{1}\right\|_{e} \leq 3\|T\|_{e}
\end{aligned}
$$

and we are done.

If $\mathcal{N}$ is a nest in $B(\mathcal{H})$, then $\pi(\mathcal{N})$ is a nest in $\mathcal{Q}$ and $\operatorname{Alg} \pi(\mathcal{N})$ is a nest subalgebra of $\mathcal{Q}$. Clearly, $\pi^{-1}(\operatorname{Alg} \pi(\mathcal{N}))=\operatorname{ET}(\mathcal{N})=\left\{A \in B(\mathcal{H}) \mid E^{\perp} A E \in \mathcal{K}\right.$ for all $\left.E \in \mathcal{N}\right\}$. Equally clearly, $\operatorname{Alg} \mathcal{N}+\mathcal{K} \subseteq \operatorname{ET}(\mathcal{N})$; whenever $\mathcal{N}$ is infinite the containment is 
proper. (By [6], $\operatorname{Alg} \mathcal{N}+K$ consists of those elements $A$ of $\operatorname{ET}(\mathcal{N})$ for which the map $E \rightarrow E^{\perp} A E$ is a strong operator topology-norm topology continuous map of $\mathcal{N}$ into $K$.)

Suppose $A$ is a positive invertible operator with spectral nest $\mathcal{N}$. By the proposition above, $\operatorname{ET}(\mathcal{N}) \subseteq B(A)$. Since $A$ can have at most countably many jumps, if $\mathcal{N}$ is uncountable the containment is proper. If $\mathcal{N}$ is countable, there exists a positive invertible operator $A$ with spectral nest $\mathcal{N}$ such that $\operatorname{ET}(\mathcal{N})=B(A)$. In general for countable nests, there exist positive invertible operators for which $B(A)$ is strictly larger than $\operatorname{ET}(\mathcal{N})$. If, however, $E$ has an immediate predecessor and an immediate successor for every $E \neq 0, I$ in $\mathcal{N}$, then $\operatorname{ET}(\mathcal{N})=B(A)$ regardless of the choice of $A$.

Finally, note that if $A_{0}$ is a positive invertible operator with spectral nest $\mathcal{N}$ and if $E \in \mathcal{N}, E \neq 0, I$, then $A=A_{0}+E$ is also a positive invertible operator with spectral nest $\mathcal{N}$. Furthermore, $A$ has a jump at $E$. Consequently, the following proposition is evident.

Proposition 2. Let $\mathcal{N}$ be a nest. Then $\operatorname{ET}(\mathcal{N})=\bigcap\{B(A): A$ is a positive invertible operator with spectral nest $\mathcal{N}\}$.

Epilogue. Every essentially triangular algebra is the inverse image under the Calkin projection of a nest-subalgebra of the Calkin algebra. This raises two related general questions:

(1) Which nest-subalgebras of the Calkin algebra lift to an essentially triangular algebra?

(2) Which nests in the Calkin algebra lift to a nest in $B(\mathcal{H})$ ?

It should be pointed out that the term "nest" is used here in two different senses. In the Calkin algebra, a nest is a totally ordered family of projections which is complete as an abstract lattice. In $B(H)$, a nest must be complete as a sublattice of the lattice of all projections in $B(\mathcal{H})$. (This is equivalent to closure in the strong operator topology.) The term chain is used to refer to any totally ordered family of projections, regardless of completeness.

Recently, Ken Davidson has made significant progress with the second question (private communication). He has proven that any countable nest in the Calkin algebra lifts to a nest in $B(\mathcal{H})$ and he has given an example of a continuous nest (i.e. a nest order isomorphic to the unit interval $[0,1])$ in the Calkin algebra which cannot be lifted to a continuous nest in $B(H)$. The nest in the example can be lifted to a chain in $B(\mathcal{H})$. The closure of this chain in the strong operator topology is the totally atomic nest whose atoms are ordered as the rational numbers. Davidson has also proven that a chain of projections in $B(\not H)$ which is order isomorphic to $[0,1]$ can be compactly perturbed to a continuous nest if and only if the completion of the atomic part of the strong operator topology closure of the chain is countable.

\section{REFERENCES}

1. N. T. Andersen, Compact perturbations of reflexive algebras, J. Funct. Anal. 38 (1980), 366-400.

2. __ Similarity of continuous nests, Bull. London Math. Soc. 15 (1983), 131-132.

3. K. Davidson, Similarity and compact perturbations of nest algebras, J. Reine Angew. Math. 348 (1984), 72-87. 
4. J. A. Deddens, Another description of nest algebras, Hilbert Space Operators, Lecture Notes in Math., Vol. 693, Springer, New York, 1978, pp. 77-86.

5. J. A. Erdos, On some ideals of nest algebras, Proc. London Math. Soc. (3) 44 (1982), 143-160.

6. T. Fall, W. Arveson and P. Muhly, Perturbations of nest algebras, J. Operator Theory 1 (1979), 137-150.

7. D. R. Larson, Nest algebras and similarity transformations, Ann. of Math. (to appear).

8. R. I. Loebl and P. S. Muhly, Analyticity and flows in von Neumann algebras, J. Funct. Anal. 29 (1978), 214-252.

9. S. C. Power, On ideals of nest subalgebras of $C^{*}$-algebras, Proc. London Math. Soc. (3) 50 (1985), 314-332.

10. J. R. Ringrose, On some algebras of operators, Proc. London Math. Soc. (3) 15 (1965), 61-83.

Department of Mathematics, Kings College London, Strand, London WC2R 2LS, ENGLAND

Department of Mathematics, University of Alabama, University, Alabama 35486 